\title{
PARAMETER ESTIMATION FOR SUMS OF CORRELATED GAMMA RANDOM VARIABLES. APPLICATION TO ANOMALY DETECTION IN INTERNET TRAFFIC.
}

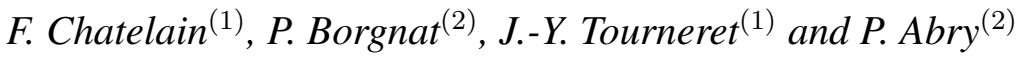 \\ (1) IRIT-ENSEEIHT, 2 rue Charles Camichel, BP 7122, 31071 Toulouse cedex 7, France \\ (2) Laboratoire de Physique, ENS Lyon, CNRS, 46 allée d'Italie, 69364 Lyon cedex 07, France \\ \{florent.chatelain,jean-yves.tourneret\}@enseeiht.fr, \{Patrice.Abry,Pierre.Borgnat\}@ens-lyon.fr
}

\begin{abstract}
A new family of distributions, constructed by summing two correlated gamma random variables, is studied. First, a simple closedform expression for their density is derived. Second, the three parameters characterizing such a density are estimated by using the maximum likelihood (ML) principle. Numerical simulations are conducted to compare the performance of the ML estimator against those of the conventional estimator of moments. Finally, a multiresolution multivariate gamma based modeling of Internet traffic illustrates the potential interest of the proposed distributions for the detection of anomalies. Aggregated times series of IP packet counts are split into adjacent non overlapping time blocks. The distribution of the resulting time series are modeled by the proposed multivariate gamma based distributions, over a collection of different aggregation levels. The anomaly detection strategy is based on tracking changes along time of the corresponding multiresolution parameters.
\end{abstract}

Index Terms - Multivariate gamma distributions, maximum likelihood estimator, Internet traffic, anomaly detection.

\section{INTRODUCTION AND PROBLEM FORMULATION}

Security issues in Internet constitute nowadays a major research topic. Notably, the statistical detection of anomalies, such as distributed denial-of-service (DDoS) attacks, has received much interest in the literature [1-4] (and references therein). Often, detection schemes are applied to aggregated times series of IP packet (or byte) counts. It is commonly accepted that such time series (consisting of positive random variables) can be well modeled by gamma distributions independently at each aggregation level. Also, such times series are strongly correlated, having both short and long range dependencies [3]. Low volume DDoS attacks, i.e., attacks that produce non noticeable changes in the volume (mean or variance) of the traffic (a relevant situation) are known to modify strongly short time correlations (cf. e.g., [2,5]). Based on such knowledge, the present contribution proposes to study traffic time series, aggregated at different levels, through a new statistical model based on multivariate gamma distributions (MGDs). More precisely, a statistical model based on sums of correlated gamma random variables is defined. The maximum likelihood estimators (MLEs) for its unknown parameters are derived. This allows us to propose a new statistical anomaly detection scheme for Internet traffic, complementing the works of $[3,4]$.

The distribution of the sums of independent gamma random variables has been studied in [6]. A generalization to sums of correlated gamma random variables has been used in [7] to assess performance of wireless communication systems over Nakagami-fading channels. However, it is a very complicated task to use the pdf in [7, Eq.
(5)] to estimate its unknown parameters. This contribution derives a new closed-form expression for the pdf of the sum of two correlated gamma random variables. This pdf is shown to be sufficiently simple to derive the MLEs for its unknown parameters.

The remainder of the text is organized as follows. Section 2 introduces MGDs and recalls some important properties of these distributions. The distribution of the sum of two gamma random variables resulting from this multivariate statistical model is then derived. Section 3 shows that the parameters of the sum of two correlated gamma random variables can be estimated by the ML method. The performance of the resulting MLE are analyzed in Section 4 by means of numerical simulations. The application to anomaly detection in Internet traffic is discussed in Section 5. Conclusions are reported in Section 6.

\section{MULTIVARIATE GAMMA BASED DISTRIBUTIONS}

\subsection{Definition and properties}

For any $q \geq 0$ and for any affine polynomial ${ }^{1} P$, a random vector $\boldsymbol{X}=\left(X_{1}, \ldots, X_{p}\right)$ is distributed according to an MGD on $\left(\mathbb{R}^{+}\right)^{d}$ with shape parameter $q$ and scale parameter $P$ (denoted as $\gamma_{q, P}$ ) if its Laplace transform (LT) is defined by (on a suitable domain of existence) $[8,9]$ :

$$
L_{\gamma_{q}, P}(\boldsymbol{z})=E\left(e^{-\sum_{i=1}^{d} X_{i} z_{i}}\right)=[P(\boldsymbol{z})]^{-q},
$$

where $E($.$) denotes the mathematical expectation. Determining the$ conditions on $q$ and $P$ such that (1) is the LT of a probability distribution defined on $\left(\mathbb{R}^{+}\right)^{d}$ is a difficult problem in the general case. However, the problem is much easier in dimension $d=2$. It can be shown that $\left[1+p_{1} z_{1}+p_{2} z_{2}+p_{12} z_{1} z_{2}\right]^{-q}$ is the LT of a probability distribution defined on $\left(\mathbb{R}^{+}\right)^{2}$ (referred to as bivariate gamma distribution) if and only if the following conditions are satisfied [9]:

$$
p_{1}>0, p_{2}>0, p_{12}>0, p_{1} p_{2}-p_{12} \geq 0 .
$$

An interesting property about MGDs is that their marginal distributions are also MGDs. For instance, by setting $z_{j}=0$ for any $j \neq i$ in (1), it can be easily seen that the marginal distribution of $X_{i}$, for $i=1, \ldots, d$ is a gamma distribution with shape parameter $q$ and scale parameter $p_{i}$, where $p_{i}$ is the coefficient of $z_{i}$ in $P(\boldsymbol{z})$. Similarly, the marginal distribution of $\left(X_{i}, X_{j}\right)$ is a bivariate gamma distribution

\footnotetext{
${ }^{1}$ A polynomial $P(\boldsymbol{z})$ with respect to $\boldsymbol{z}=\left(z_{1}, \ldots, z_{d}\right)$ is affine if for any $j=1, \ldots, d$, the one variable polynomial $z_{j} \mapsto P(\boldsymbol{z})$ has the form $A z_{j}+B$, where $A$ and $B$ are polynomials with respect to the $z_{i}$ 's with $i \neq j$.
} 
with shape parameter $q$ and its scale parameter is the affine polynomial $P\left(z_{i}, z_{j}\right)=1+p_{i} z_{i}+p_{j} z_{j}+p_{i j} z_{i j}$. Note that the definition (1) implies that all marginal distributions have the same shape parameter $q$. The reader is invited to consult [8-10] for having more properties regarding these distributions. In particular, closed form expressions for the moments, the correlation coefficient and the pdf of bivariate gamma distributions have been derived. The next section derives a simple closed form expression for the pdf of $X_{i}+X_{j}$ which is not available in the references above. We will explain later that this distribution is interesting to detect anomalies in internet traffic.

\subsection{Distribution of $X_{i}+X_{j}$}

Theorem 2.1. For an MGD with LT (1), the pdf of $Y_{i j}=X_{i}+X_{j}$ denoted as $p\left(y_{i j}\right)$ is

$$
p\left(y_{i j}\right)=\frac{\left[2 \sqrt{\pi} y_{i j}^{2 q-1}\right] e^{-\frac{2 q y_{i j}}{m(1-r)}}}{(1-r)^{q}(m / q)^{2 q} \Gamma(q)} f_{\frac{q+1}{2}}\left(\frac{c y_{i j}^{2}}{4}\right) I_{\mathbb{R}}+\left(y_{i j}\right),
$$

where $r=\frac{p_{i} p_{j}-p_{i j}}{p_{i} p_{j}}, m=q\left(p_{i}+p_{j}\right), c=\frac{4 q^{2} r}{m^{2}(1-r)^{2}}, p_{i}, p_{j}, p_{i j}$ are the coefficients of $z_{i}, z_{j}, z_{i} z_{j}$ in the affine polynomial $P(z)$ defined in (1), $\Gamma(z)$ is the Gamma function, $f_{q}(z)=\sum_{k=0}^{\infty} \frac{z^{k}}{\Gamma(k+1) \Gamma(k+q)}$ and $I_{\mathbb{R}^{+}}($.$) is the indicator function on \mathbb{R}^{+}$.

Proof. The proof given in this paper is based on the properties of natural exponential families ${ }^{2}$. The LT of $Y_{i j}=X_{i}+X_{j}$ can be written

$$
L_{Y_{i j}}(z)=E\left(e^{-z Y_{i j}}\right)=\left[1+\left(p_{i}+p_{j}\right) z+p_{i j} z^{2}\right]^{-q} .
$$

The parameterizations $(m, r, q)$ and $(\theta, c, q)$ with $\theta=(2 q) /[m(1-$ $r)$ ] lead to the following results:

$$
\begin{aligned}
L_{Y_{i j}}(z) & =\left(1+\frac{m}{q} z+\frac{(1-r) m^{2}}{4 q^{2}} z^{2}\right)^{-q}, \\
& =\left(1+2 \frac{\theta}{\theta^{2}-c} z+\frac{1}{\theta^{2}-c} z^{2}\right)^{-q} .
\end{aligned}
$$

This last expression shows that the LT of $Y_{i j}$ can be expressed as

$$
L_{Y_{i j}}(z)=\frac{L_{\mu}(\theta+z)}{L_{\mu}(\theta)}
$$

where $L_{\mu}(\theta)=\left(\theta^{2}-c\right)^{-q}$ is the LT of the so-called generating measure of the natural exponential family. By noting that the LT of $e^{-\theta y_{i j}} \mu\left(y_{i j}\right)$ is $L_{\mu}(\theta+z)$, a direct consequence of (4) is that the pdf of $Y_{i j}$ can be written

$$
p\left(y_{i j}\right)=\frac{1}{L_{\mu}(\theta)} e^{-\theta y_{i j}} \mu\left(y_{i j}\right) .
$$

The pdf of $Y_{i j}$ can then be determined by (5) providing the generating measure $\mu\left(y_{i j}\right)$ is known. The last part of the proof consists of determining the generating measure $\mu\left(y_{i j}\right)$ with LT

$$
L_{\mu}(\theta)=\left(\theta^{2}-c\right)^{-q}=\frac{1}{\theta^{2 q}} \frac{1}{\left(1-\frac{c}{\theta^{2}}\right)^{q}} .
$$

It has been indicated before that the coefficients $p_{i}, p_{j}$ and $p_{i j}$ of the bivariate gamma distribution of $\left(X_{i}, X_{j}\right)$ satisfy the conditions (2). As a consequence

$$
\frac{c}{\theta^{2}}=r=\frac{p_{i} p_{j}-p_{i j}}{p_{i} p_{j}} \in[0,1[.
$$

\footnotetext{
${ }^{2}$ The authors are very grateful to Gérard Letac for the many interesting discussions regarding MGDs and natural exponential families.
}

This allows one to use the following expansion for $z=\frac{c}{\theta^{2}}$

$$
\frac{1}{(1-z)^{q}}=\sum_{k=0}^{\infty} \frac{(q)_{k}}{\Gamma(k+1)} z^{k}
$$

where $(q)_{k}=q(q+1) \ldots(q+k-1)=\frac{\Gamma(q+k)}{\Gamma(q)}$ is the Pochhammer symbol $[11$, p. 256]. After replacing the expansion above in (6), the following result can be obtained:

$$
L_{\mu}(\theta)=\sum_{k=0}^{\infty} \frac{(q)_{k}}{\Gamma(k+1)} \frac{c^{k}}{\theta^{2 q+2 k}}
$$

By using the classical definition of the gamma function $\Gamma(p)=$ $\theta^{-p} \int_{0}^{\infty} e^{-\theta x} x^{p-1} d x$, the following result can be obtained

$$
L_{\mu}(\theta)=\sum_{k=0}^{\infty} \frac{(q)_{k} c^{k}}{\Gamma(k+1)} \int_{0}^{\infty} e^{-\theta x} \frac{x^{2 q+2 k-1}}{\Gamma(2(q+k))} d x=\int_{0}^{\infty} e^{-\theta x} \mu(x),
$$

with

$\mu(x)=\sum_{k=0}^{\infty} \frac{(q)_{k} c^{k}}{\Gamma(k+1)} \frac{x^{2 q+2 k-1}}{\Gamma[2(q+k)]}=\sqrt{\pi} \frac{(x / 2)^{2 q-1}}{\Gamma(q)} f_{q+1 / 2}\left(c(x / 2)^{2}\right)$.

This concludes the proof.

It is interesting to note that (3) is simpler that the expression of $p\left(y_{i j}\right)$ derived in [7, eq. (5)] which requires to evaluate products of infinite series. Note also that the generalization of (3) to sums of more than two gamma random variables is not straightforward. The next section shows that the expression of $p\left(y_{i j}\right)$ obtained in (3) can be used to derive the MLEs of the unknown parameters characterizing the distribution of $X_{i}+X_{j}$.

\section{PARAMETER ESTIMATION}

The pdf of the sum of correlated gamma variates derived in (3) is characterized by the parameter vector $\boldsymbol{\theta}=(m, r, q)$, where $m$ is the mean of the distribution (i.e. $\left.m=E\left[X_{i}\right]\right)$ and $r$ is the correlation coefficient of $\left(X_{i}, X_{j}\right)$ [10]. This section addresses the problem of estimating the unknown parameter vector $\boldsymbol{\theta}$ from $n$ independent vectors $Y_{1}, \ldots, Y_{n}$ having the same pdf (3).

\subsection{Maximum likelihood estimation}

The MLE of $\boldsymbol{\theta}$ is obtained by maximizing the joint log-likelihood

$$
\begin{gathered}
l(\boldsymbol{y} ; \boldsymbol{\theta})=n \log \frac{\sqrt{\pi}}{\Gamma(q)}-n q \log \left[(1-r) \frac{m^{2}}{q^{2}}\right]-n \frac{2 q \bar{y}_{n}}{m(1-r)} \\
+\left(q-\frac{1}{2}\right) \sum_{i=1}^{n} \log y_{i}+\sum_{i=1}^{n} \log f_{q+1 / 2}\left(\frac{c y_{i}^{2}}{4}\right) .
\end{gathered}
$$

where $\boldsymbol{y}=\left(y_{1}, \ldots, y_{n}\right)$ and $\bar{y}_{n}=\frac{1}{n} \sum_{i=1}^{n} y_{i}$. By differentiating $l(\boldsymbol{y} ; \boldsymbol{\theta})$ with respect to $m$ and $r$, the following results are obtained

$$
\begin{aligned}
& -\frac{2 n q}{m}+\frac{2 n q \bar{y}_{n}}{m^{2}(1-r)}-\frac{2 q^{2} r}{m^{3}(1-r)} C(\boldsymbol{y}, c, q)=0 \\
& \frac{n q}{1-r}-\frac{2 n q \bar{y}_{n}}{m(1-r)^{2}}+\frac{q^{2}}{m^{2}} \frac{1+r}{(1-r)^{3}} C(\boldsymbol{y}, c, q)=0,
\end{aligned}
$$


where

$$
C(\boldsymbol{y}, c, q)=\sum_{i=1}^{n} y_{i}^{2} \frac{f_{q+\frac{3}{2}}\left(\frac{c y_{i}^{2}}{4}\right)}{f_{q+\frac{1}{2}}\left(\frac{c y_{i}^{2}}{4}\right)} .
$$

Eliminating $C(\boldsymbol{y}, c, q)$ in (8) and replacing its expression in (7) yields the following trivial MLE for parameter $m$ :

$$
\widehat{m}_{\mathrm{MV}}=\overline{\boldsymbol{y}}_{n} .
$$

The estimation of $r$ and $q$ is more complicated. After replacing $\widehat{m}_{\mathrm{MV}}$ in the log-likelihood function $l(\boldsymbol{y} ; \boldsymbol{\theta})$, the MLE of $(r, q)$ can be obtained by maximizing the following nonlinear function

$$
\begin{aligned}
g(r, q)= & -n q \log \left[\frac{\overline{\boldsymbol{y}}_{n}^{2}}{4 q^{2}}(1-r)\right]-2 n q(1-r) \\
& +\sum_{i=1}^{n} \log f_{q+\frac{1}{2}}\left(\widehat{c}_{\mathrm{MV}} \frac{y_{i}^{2}}{4}\right)-n \log \Gamma(q),
\end{aligned}
$$

with $\widehat{c}_{\mathrm{MV}}=\frac{4 q^{2} r}{\overline{\boldsymbol{y}}_{n}^{2}(1-r)^{2}}$. This maximization is conducted by using a Newton-Raphson procedure initialized by a moment estimator of $(r, q)$. This initialization is described in the next section.

\subsection{Moment estimator of $(r, q)$ for MLE initialization}

This section derives a moment estimator of $(r, q)$ which will be used to initialize the Newton-Raphson procedure for maximizing the nonlinear function $g(r, q)$. The moments of $Y_{i}$ can be computed from the moments of MGDs derived for instance in [10]. For instance, the following results will be useful in this paper: $E\left[Y_{i}\right]=m$, $\operatorname{Var}\left[Y_{i}\right]=\frac{m^{2}}{2 q}(1+r)$ and $E\left[\left(Y_{i}-E\left[Y_{i}\right]\right)^{3}\right]=\frac{m^{2}}{2 q^{2}}(-1+3 r)$. The first equality shows that the MLE of $m$ is also a moment estimator computed from its first order moment. The other two equalities show that a moment estimator of $(r, q)$ can be obtained as the solution of the following system

$$
\begin{aligned}
& \widehat{r}_{\mathrm{Mo}}=\frac{1}{2 \widehat{q}_{\mathrm{Mo}}^{2}}\left[-\frac{\bar{y}_{n}^{2}}{2}+\frac{\widehat{q}_{\mathrm{Mo}}}{n} \sum_{i=1}^{n}\left(y_{i}-\bar{y}_{n}\right)^{2}\right], \\
& \frac{2 \widehat{q}_{\mathrm{Mo}}^{2}}{n} \sum_{i=1}^{n}\left(y_{i}-\bar{y}_{n}\right)^{3}-3 \widehat{q}_{\mathrm{Mo}} \frac{\bar{y}_{n}}{n} \sum_{i=1}^{n}\left(y_{i}-\bar{y}_{n}\right)^{2}+5 \bar{y}_{n}^{3}=0 .
\end{aligned}
$$

Note that $\widehat{q}_{\text {Mo }}$ satisfies a second order equation whose number of positive solutions depends on the sign of the estimated third order moment $\widehat{\mu}_{3}=\frac{1}{n} \sum_{i=1}^{n}\left(y_{i}-\bar{y}_{n}\right)^{3}$. More precisely, Eq. (10) has two positive solutions when $\widehat{\mu}_{3}>0$ and a single positive solution when $\widehat{\mu}_{3}<0$. In the former case, prior information regarding the value of $q$ is required to determine the appropriate solution.

\section{SIMULATION RESULTS FOR SYNTHETIC SIGNALS}

The MLE for parameter $m$ defined previously is unbiased, convergent and efficient. Thus the performance of this estimator is fully controlled by its variance defined by

$$
\operatorname{var}\left(\widehat{m}_{\mathrm{MV}}\right)=\frac{m^{2}(1+r)}{2 n q} .
$$

This section studies the mean square errors (MSEs) of the MLE for the parameter vector $(r, q)$. Note that this MSE cannot be expressed in closed form as for parameter $m$. Figures 1 and 2 display the MSEs of $\widehat{q}_{\mathrm{MV}}$ and $\widehat{r}_{\mathrm{MV}}$ (log scale) as a function of the sample size $\log _{10} n$ for 10000 Monte Carlo (MC) runs (the true parameters are $r=0.8$, $m=2$ and $q=0.5$ ). These MSEs are compared with the corresponding Cramer Rao lower bounds (CRLB) (estimated by Monte Carlo averages) and with the moment estimator errors. The MSEs of the MLE are clearly close to the CRLBs for large sample sizes. Significant improvement in estimation performance is obtained when using the ML method with respect to the method of moments. It is interesting to note that a similar behavior for these estimators has been observed for bivariate gamma distributions [10].

\section{ANOMALY DETECTION IN INTERNET TRAFFIC}

Internet information flows are commonly analyzed in terms of aggregated time series, $X_{\Delta_{0}}(k)$, consisting of the counts of IP packets per time bin of size $\Delta_{0}$ as a function of time $t=k \Delta_{0}$, for $k=1, \ldots, n$. A challenge in nowadays Internet monitoring amounts to detecting anomalous (potentially aggressive) behaviors in the time course of series $X_{\Delta_{0}}(k)$. To this end, several statistical detection schemes were proposed (cf. e.g., [3] and references therein). The approach developed in [3] indicates that the marginal distribution of $X_{\Delta_{0}}(k)$ is satisfactory modeled by a gamma law, while its covariance is well described with an $\operatorname{ARFIMA}(\phi, d, \theta)$ (where $d$ is related to the long memory Hurst parameter $H=d+1 / 2$, while $\phi$ and $\theta$ denote the ARMA parameters). Elaborating on intuitions developed in [3,4], the proposed anomaly detection scheme is based on a multiresolution multivariate gamma modeling, whose parameters characterize the occurrence (or not) of anomalous behaviors along time.

More precisely, let $X_{\Delta_{j}}(k)=X_{\Delta_{j-1}}(2 k)+X_{\Delta_{j-1}}(2 k+1)$ stands for the multiresolution description of the traffic aggregated at levels $\Delta_{j}=2^{j} \Delta_{0}$, for time $k \in\left[1,2^{-j}\right]$ and scales $j=1, \ldots, J$. The time series $X_{\Delta_{0}}(k)$ is split into $L$ non overlapping consecutive series $\left\{X_{\Delta_{0}}^{(l)}(k), l=1, \ldots, L\right\}$ for $k=1, \ldots, n$. The proposed strategy estimates the parameters $\hat{\boldsymbol{\theta}}_{j}^{(l)}=\left(\hat{m}_{j}^{(l)}, \hat{r}_{j}^{(l)}, \hat{q}_{j}^{(l)}\right)$ of the pdf (3) for $X_{\Delta_{j}}^{(l)}$ (these parameters are the mean of $X_{\Delta_{j}}(k)$, the correlation coefficient between $X_{\Delta_{j-1}}(k)$ and $X_{\Delta_{j-1}}(k+1)$ and the shape parameter of the pdf). Our characterization scheme tracks changes along time $l$ of $\hat{q}_{j}^{(l)}, \hat{r}_{j}^{(l)}$ as functions of the aggregation level $j$. The intuitions beyond such a scheme are as follows: modern malicious anomalies no longer correspond to volume (mean or variance) changes but rather to slight modifications in the correlations of the sequence $X_{\Delta_{0}}(k)$. Theoretical analysis of the statistics of $X_{\Delta_{j}}$ show that this should significantly impact the evolution of $q_{j}$ and $r_{j}$ with respect to $j$.

Let us illustrate this scheme at work on a simulated example. Consider a synthetic aggregated time series $X_{\Delta_{0}}(k)\left(\right.$ with $n=2^{15}$ ) whose marginal distribution is a gamma distribution $\gamma_{2,3}$ and with an $\operatorname{ARFIMA}(\phi=0.01, d=0.3, \theta=0.7)$ covariance (these values are relevant for Internet traffic). A short duration $\left(n_{A}=2^{11}\right)$ anomaly is superimposed to $X_{\Delta_{0}}$. In consistence with empirical observations, the anomaly is independent of $X_{\Delta_{0}}$. It is chosen so as to have little impact on the overall marginal distribution at $\Delta_{0}$, but with its own specific covariance structure given by an $\operatorname{ARMA}\left(\phi_{A}=\right.$ $0.9, \theta_{A}=0$ ) model. It is added to $X_{\Delta_{0}}$ at a given arbitrary position. Note that the peak intensity of the anomaly is around $25 \%$ in packet count, has no impact on the mean value of the traffic, and is completely invisible by eye inspection. The estimated $\hat{q}_{j}^{(l)}, \hat{r}_{j}^{(l)}$ obtained with $L=4$ time series are shown in Figs. 3(a) and 3(b) as functions of $j$, where $L=4$ is chosen for clarity of the plots. These plots clearly show that the estimated functions $\hat{q}_{j}^{(l)}, \hat{r}_{j}^{(l)}$ are mostly consistent from one time window to another in absence of anomaly (solid red curve with circles). On the contrary, the time window 
that contains the superimposed anomaly yields clear changes in the estimated functions $\hat{q}_{j}, \hat{r}_{j}$ (solid black curve with diamonds). The presence of an anomaly mostly affects $q_{j}$ at the coarsest aggregation levels in agreement with $[3,4]$. In addition, the proposed analysis shows dramatic changes in $r_{j}$, occurring mostly at the finest aggregation levels, and related to the modification of the short time correlations induced by the anomaly. This discrepancy can be quantified making use of statistical distances (such as e.g., Mahalanobis distance) and can serve as a basis for the design of more sensitive statistical detection tests. Such tests are currently under investigation.

\section{CONCLUSIONS}

A simple closed-form expression for the pdf of sums of correlated gamma random variables has been obtained. This closed-form pdf has been used to derive maximum likelihood estimators for the corresponding parameters. The potential interest of using sums of correlated gamma random variables for anomaly detection in Internet traffic has been illustrated. The detailed definition and performance of anomaly detection schemes are being investigated. The results obtained here can also be used in a number of other applications involving sums of gamma random variables including wireless communications [7] or image processing [12].

\section{REFERENCES}

[1] D. Moore, G.M. Voelker, and S. Savage, "Inferring Internet denialof-service activity," in Usenix Security Symposium, Washington D.C., USA, Aug. 2001, pp. 9-22.

[2] A. Hussain, J. Heidemann, and C. Papadopoulos, "A framework for classifying denial of service attacks," in SIGCOMM, Kalsruhe, Germany, Aug. 2003, pp. 99-110.

[3] A. Scherrer, N. Larrieu, P. Owezarski, P. Borgnat, and P. Abry, "Non gaussian and long memory statistical characterisations for internet traffic with anomalies," IEEE Trans. Dependable Secur. Comput., vol. 4 , no. 1, pp. 56-70, Jan. 2007.

[4] G. Dewaele, K. Fukuda, P. Borgnat, P. Abry, and K. Cho, "Extracting hidden anomalies using sketch and non gaussian multiresolution statistical detection procedures," in SIGCOMM Workshop LSAD, Kyoto, Japan, Aug. 2007, pp. 99-110.

[5] P. Barford, J. Kline, D. Plonka, and A. Ron, "A signal analysis of network traffic anomalies," in $I M W$, Marseille, France, Nov. 2002, pp. $71-82$.

[6] P. G. Moschopoulos, "The distribution of the sum of independent gamma random variables," Ann. Inst. Statist. Math. (Part A), vol. 37 pp. 541-544, 1985.

[7] M. S. Alouini, A. Abdi, and M. Kaveh, "Sum of gamma variates and performance of wireless communication systems over Nakagami fading channels," IEEE Trans. on Veh. Technol., vol. 50, no. 6, pp. 1471-1480, Nov. 2001.

[8] S. Bar Lev, D. Bshouty, P. Enis, G. Letac, I. L. Lu, and D. Richards, "The diagonal natural exponential families and their classification," $J$. of Theoret. Probab., vol. 7, no. 4, pp. 883-928, Oct. 1994.

[9] P. Bernardoff, "Which multivariate Gamma distributions are infinitely divisible?," Bernoulli, vol. 12, no. 1, pp. 169-189, Feb. 2006

[10] F. Chatelain, J.-Y. Tourneret, A. Ferrari, and J. Inglada, "Bivariate gamma distributions for image registration and change detection," IEEE Trans. Image Processing, vol. 16, no. 7, pp. 1796-1806, July 2007.

[11] M. Abramowitz and I. Stegun, Handbook of Mathematical Functions, Dover, New York, 1972.

[12] P. Réfrégier, Julien Fade, and Muriel Roche, "Estimation precision of the degree of polarization from a single intensity image," Optics Letters, vol. 32, no. 7, pp. 739-741, April 2007.

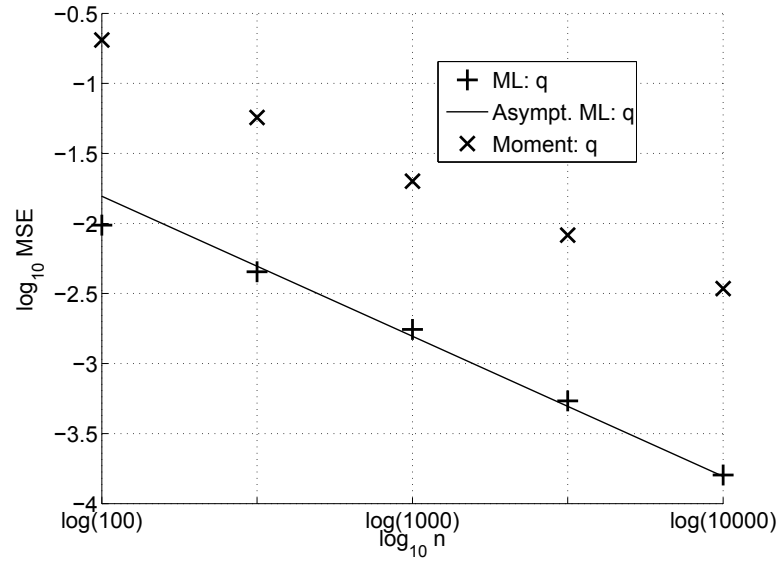

Fig. 1. $\log _{10}(\mathrm{MSE})$ of $\widehat{q}_{\mathrm{MV}}$ versus $\log _{10}(n)$ (10000 MC runs).

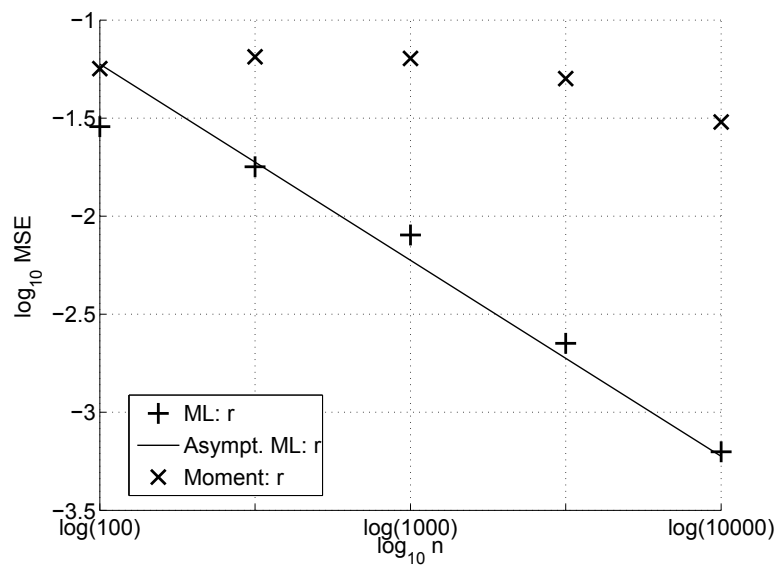

Fig. 2. $\log _{10}(\mathrm{MSE})$ of $\widehat{r}_{\mathrm{MV}}$ versus $\log _{10}(n)$ (10000 MC runs).

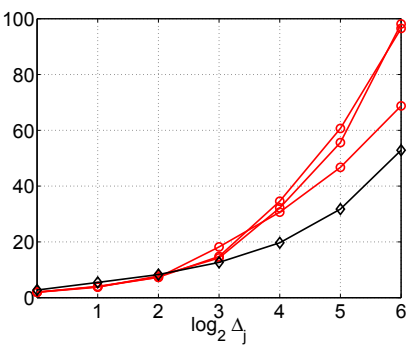

(a) Parameter $\hat{q}_{j}$.

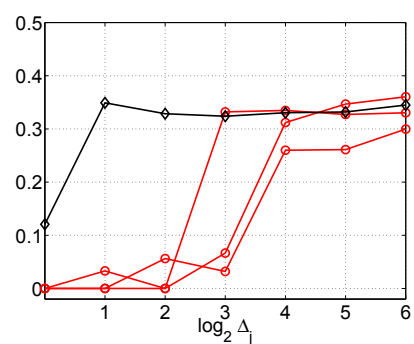

(b) Parameter $\hat{r}_{j}$.
Fig. 3. Estimated parameters $\hat{q}_{j}$ (left) and $\hat{r}_{j}$ (right) as a function of the (log of the) aggregation level. Red 'o' correspond to time windows without any anomaly, while black ' $\diamond$ ' correspond to the window affected by the anomaly. 